American Journal of Infectious Diseases 6 (4): 103-106, 2010

ISSN 1553-6203

(C) 2010 Science Publications

\title{
Miliary Tuberculosis: A Case Report
}

\author{
${ }^{1}$ Zhinous Bayat Makoo, ${ }^{2}$ Roshanak Bayat Makoo and ${ }^{3}$ Omid Mashrabi \\ ${ }^{1}$ Department of Infectious Disease Specialist, \\ Infectious Diseases and Tropical Medicine Research Center, \\ Tabriz University of Medical Sciences, Tabriz, Iran \\ ${ }^{2}$ MSC of Biochemistry, Faculty of Medicine, \\ Islamic Azad University Tabriz Branch, Iran \\ ${ }^{3}$ Department of General Physician, Faculty of Medicine, \\ Tabriz University of Medical Sciences, Tabriz, Iran
}

\begin{abstract}
Problem statement: During the last years it is more than evident that the prevalence of pulmonary and extrapulmonary Tuberculosis (TB), mainly in western European countries, has risen significantly. The aetiology of this phenomenon is multifactorial. Miliary tuberculosis is a form of tuberculosis that is characterized by millet-like seeding of TB bacilli in the lung, as evidenced on chest radiography. Approach: A 17-year-old woman with headache, nausea-vomiting and fever of two months before. The patient's symptoms exacerbate and had weight that referred to the emergency department with severe headache and frequently vomiting. Bilateral coarse ceracel without wheezing. In primary CXR, diffuse reticulonodular two lungs with opened with pleural open angles. In thoracic HRCT images, diffuse micronodular lesions in throughout the lung Parenchyma, peribronchovascular thickening in parahilar areas and significant increases in the thickness of several upper lobe bronchus. In brain MRI, multiple micronodular lesions with probably miliary TB were reported. AFB of BAL sample was $1^{+}$and cytology of BAL was negative for malignancy. HIV Ab, HBS Ag, HCV Ab and IgM HAV were negative. U/A, U/C B/C was normal. Conclusion: Results of BAL culture were positive for TB and Granulomatous inflammation, surrounded by mile lymphocytic infiltrate, with central necrosis, suggestive of TB were reported in endobronchial biopsy.
\end{abstract}

Key words: Extrapulmonary tuberculosis, miliary tuberculosis, diagnosis, Human Immunodeficiency Virus (HIV), primary CXR, thoracic HRCT images, HCV Ab, phenomenon is multifactorial, endobronchial biopsy, granulomatous inflammation

\section{INTRODUCTION}

During the last years it is more than evident that the prevalence of pulmonary and extrapulmonary Tuberculosis (TB), mainly in western European countries, has risen significantly. The etiology of this phenomenon is multifactorial (Mehta et al., 1991; Yao and Sartoris, 1995).

Miliary tuberculosis is a form of tuberculosis that is characterized by millet-like seeding of TB bacilli in the lung, as evidenced on chest radiography (Moon, 1997).

Miliary TB may occur in an individual organ (very rare, $<5 \%$ ) or may affect any number of organs throughout the whole body ( $>90 \%$ ), including brain, lungs, liver and spleen. It is a complication of $1-3 \%$ of all TB cases (Sharma et al., 2005).
Miliary tuberculosis accounts for $1-2 \%$ of patients with tuberculosis. Predisposing factors for development of military tuberculosis include advanced age, Human Immunodeficiency Virus (HIV) infection, malnutrition, diabetes mellitus, chronic renal failure, organ transplantation, corticosteroids, silicosis, connective tissue disease, immunosuppressive therapy and pregnancy (Golden and Vikram, 2005; Zaeeter et al.,2010).

Case report: A 17-year-old woman with headache, nausea-vomiting and fever of two months before that underwent several ineffective treatments and in this period, the patient's symptoms exacerbate and had weight that referred to the emergency department with severe headache and frequently vomiting and for More reviews, will be admitted in the Infectious disease ward.

Corresponding Author: Mashrabi Omid, Faculty of Medicine, Tabriz University of Medical Sciences, Tabriz, Iran Tel: +98-9144049694/+98-4226226106 
She had headache, weakness and lethargy, nausea and vomiting, anorexia, weight loss, dry cough and muscle pain. In physical examination, alert, ill and nonToxic, BT, BP, PR, RR and BW were 37.2, 112/76, $107,32,45 \mathrm{~kg}$ and O2sat $=97 \%$ with nasal oxygen, respectively.

No Lymphadenopathy was dehydrated mucus Supraclavicular, was Axillary and inguinal.

In Lung examination found bilateral coarse ceracel without wheezing. PMH and FH of patient were negative. Patient was admitted by pneumonia and meningitis rule out.

LPhad done in emergency ward that's has 4 lymphocyte, normal protein and low glucose.

In primary CXR, diffuse Reticulonodular two lungs with opened with pleural open angles. Brain CT scan was normal. After admission, headache, vomiting and fever $\left(40^{\circ} \mathrm{C}\right)$ were exacerbated. In complementary evaluation, rheumatologic tests, Wright and Coombs Wright were negative and second LP had similar primary LP results (Table 2). In thoracic HRCT images, diffuse micronodular lesions in throughout the lung Parenchyma, peribronchovascular thickening in parahilar areas and significant increases in the thickness of several upper lobe bronchus. In brain MRI, multiple micronodular lesions with probably miliary TB were reported.

There were no eye tubercles and the morning urine smear in three times and CSF smear in two times were negative for TB. PPD was negative and the abdominal and pelvic ultrasound and lymph nodes were all normal. Vegetation was not seen in echocardiography and Bronchoscopy, hypervascular mucosa was seen in distal to trachea.

AFB of BAL sample was 1+ and cytology of BAL was negative for malignancy. HIV Ab, HBS Ag, HCV Ab and IgM HAV were negative. U/A, U/C B/C was normal.

In spiral CT scan of thorax, diffuse Hematogen TB type lesions and Micro-adenopathy of sub cranial and right Para pharyngeal were found.

4 days after admission, because of patient deplorability and miliary TB possibility, empirical 4 drug TB treatment such as Rifampicin $400 \mathrm{mg}$, pirazinamide $1 \mathrm{~g}$, Ethambutol $800 \mathrm{mg}$ and Isoniazid 300 and Pyridoxine started and during the three days after starting treatment headache improved and fever, nausea and vomiting decreased.

Fourth day of anti-TB treatment, the patient suffered abdominal pain, nausea and frequent that in physical examination, epigastric and RUG tenderness was found and significant increase was observed in liver enzymes that in left day, increased ALT up to 1200 and AST up to 386.
With drug hepatitis, Isoniazid, Pirazinamide, Rifampicin withdrawn and replaced with streptomycin injections $750 \mathrm{mg}$ and Ofloxacin $800 \mathrm{mg}$ daily.

During the following days and controlling liver enzyme and due to decreasing liver enzyme levels, low dose Isoniazid started and at lost patients was discharged with partial improvement with Isoniazid 300 mg, Rifampicin $430 \mathrm{mg}$, Ethambutol $800 \mathrm{mg}$, streptomycin $730 \mathrm{mg}$ and vit B6.

Results of BAL culture were positive for TB and Granulomatous inflammation, surrounded by mile lymphocytic infiltrate, with central necrosis, suggestive of TB were reported in endobronchial biopsy.

Laboratory finding and CSF fluid analysis of patient were showed in Table 1 and 2.

Chest CT_Sacn and Smear lung biopsy were showed in Fig. 1 and 2.

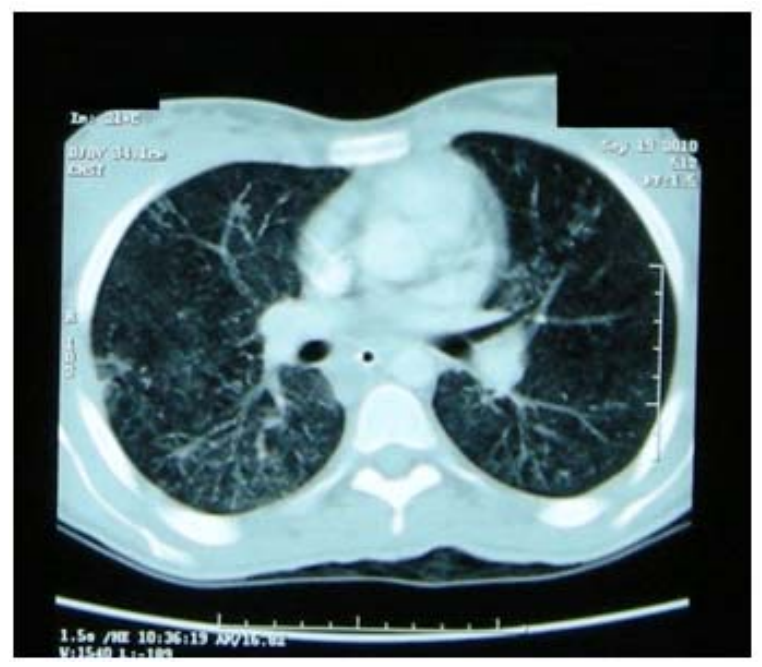

Fig. 1: Chest CT_Sacn of patient

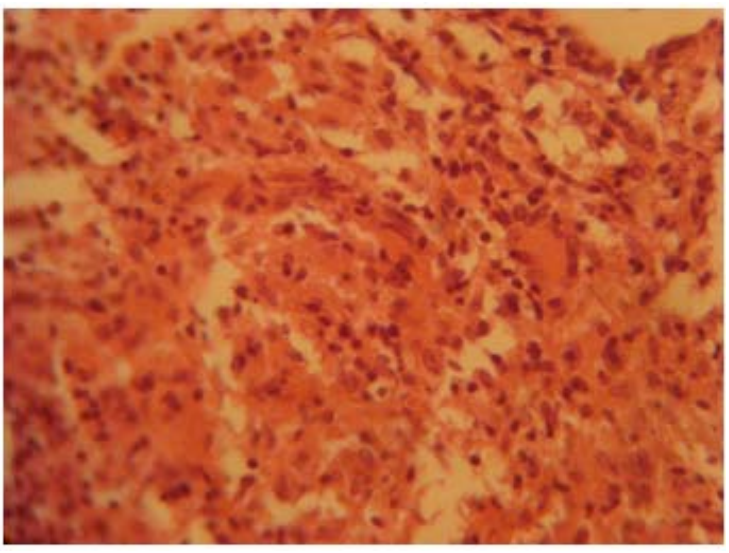

Fig. 2: Smear of lung biopsy of patient 
Am. J. Infect. Dis., 6 (4): 103-106, 2010

Table 1: Laboratory finding of patient

\begin{tabular}{|c|c|c|c|c|c|c|}
\hline & 1st day & 1 week later & 2 week later & 3 week later & 4 week later & $\begin{array}{l}2 \text { week } \\
\text { after discharge }\end{array}$ \\
\hline WBC & 5630.00 & 3910.0 & 4000.00 & 5450.0 & - & 5950.000 \\
\hline $\mathrm{Hb}$ & 10.80 & 10.1 & 10.20 & 11.7 & - & 11.600 \\
\hline HCT & 36.90 & 34.1 & 31.00 & 39.1 & - & 42.000 \\
\hline PLT & 264000.00 & 246000.0 & 316000.00 & 387000.0 & - & 543000.000 \\
\hline SGOT & 90.00 & 676.0 & 64.00 & 65.0 & 65.0 & 46.000 \\
\hline SGOP & 49.00 & 435.0 & 117.00 & 32.0 & 37.0 & 45.000 \\
\hline ALP & 172.00 & 249.0 & 273.00 & 252.0 & 282.0 & 305.000 \\
\hline PT & 15.00 & 14.0 & 14.00 & 12.0 & 12.5 & 12.500 \\
\hline PTT & 38.00 & 35.0 & 35.00 & 28.0 & 39.0 & 36.000 \\
\hline INR & 1.70 & 1.4 & 1.40 & 1.0 & 1.1 & 1.000 \\
\hline Bilirubin total & - & - & 0.70 & 0.9 & - & 0.350 \\
\hline Bil Direct & - & - & 0.30 & 0.3 & - & 0.200 \\
\hline Amylase & - & - & 85.00 & - & - & - \\
\hline Lipase & & - & 49.00 & - & - & - \\
\hline Aldolase & - & - & 13.00 & - & - & - \\
\hline CPK & - & - & 5.37 & - & - & - \\
\hline Ceruloplasmin & 0.45 & - & - & - & - & 0.669 \\
\hline Albumin & 4.80 & - & - & - & - & - \\
\hline ACE & 90.00 & 143.0 & - & - & - & 92.000 \\
\hline ESR & $13 / 30$ & - & - & - & - & 48.000 \\
\hline CRP & $3+$ & - & - & - & - & 9.000 \\
\hline Urine CU & - & 48.0 & - & - & - & - \\
\hline $\mathrm{Fe}$ & 27.00 & - & - & - & - & - \\
\hline $\mathrm{Ca}$ & 8.30 & - & 8.20 & - & - & - \\
\hline BS & 94.00 & - & 62.00 & - & - & - \\
\hline $\mathrm{Cr}$ & 0.60 & - & 0.70 & - & - & 0.600 \\
\hline Urea & 23.00 & - & - & - & - & - \\
\hline Ferritin & 32.00 & - & - & - & - & - \\
\hline TIBC & 258.00 & - & - & - & - & - \\
\hline
\end{tabular}

Table 2: CSF fluid analysis of patient

\begin{tabular}{lccc}
\multicolumn{4}{c}{ Table 2: CSF fluid analysis of patient } \\
& CSF fluid analysis & \\
& 1st day & 3rd day & 4th day \\
\hline WBC & 4 & 0.0 & 7 \\
Neutrophil & 0 & 0.0 & 0 \\
Lymphocyte & $100 \%$ & $100.0 \%$ & $100 \%$ \\
RBC & 5500 & 1100.0 & 320 \\
Protein & 40 & 41.8 & 46 \\
LDH & 100 & 93.0 & 112 \\
GLC & 20 & 11.0 & 28 \\
ADA & - & 5.0 & - \\
Wright (CSF) & & Neg & \\
Coombs Wright (CSF) & Neg & Neg & Neg \\
Smear & Neg & Neg & Neg \\
Culture & & &
\end{tabular}

\section{CONCLUSION}

Miliary tuberculosis is a potentially lethal form of tuberculosis resulting from massive lymphohaematogeneous dissemination of Mycobacterium tuberculosis bacilli (Sharma et al., 2005).

Diagnosis of extrapulmonary tuberculosis is often difficult. Although positive chest radiographic findings or a positive tuberculin skin test supports the diagnosis, negative results do not exclude extrapulmonary tuberculosis (Engin et al., 2000).

Miliary TB is common disease in areas where TB is endemic. It is the widespread dissemination of
Mycobacterium tuberculosis from hematogenous spread (Dagli et al., 2009).

Miliary TB may mimic many diseases and Symptoms including fever, night sweats, weight loss, hematologic abnormalities, cough, pleurisy, dyspnea and hemoptysis are not necessarily specific. In some case series, up to $50 \%$ of cases are not diagnosed antemortem (Sharma et al., 2005).

Our case also had fever, night sweats, weight loss, cough, pleurisy, dyspnea.

Tuberculosis (TB) is a serious disease of global importance, with a rising incidence in the developed world in recent years (Carrol et al., 2001).

Novel diagnostic modalities such as adenosine deaminase levels and polymerase chain reaction method can be useful in certain forms of extrapulmonary tuberculosis (Moon, 1997).

The infection is characterized by the appearance of numerous small nodular lesions that resemble millet seeds on chest radiography (Dagli et al., 2009).

CT scanning of the chest may help to better define abnormalities in patients with vague findings on chest radiography (Dagli et al., 2009).

Also, in our case, in thoracic HRCT images, diffuse micronodular lesions in throughout the lung 
Parenchyma, peribronchovascular thickening in parahilar areas and significant increases in the thickness of several upper lobe bronchus and in brain MRI, multiple micronodular lesions with probably miliary TB were reported.

Clinical manifestations are nonspecific and include fever, chills, night sweats, weight loss and abnormal aminotransferases (Golden and Vikram, 2005; Sharma et al., 2005; Lim and Chong, 1999).

Examination of the sputum, gastric washing, bronchoalveolar lavage and blood cultures may be necessary to establish the diagnosis. Miliary tuberculosis is a fatal disease if not treated. Antituberculosis treatment is the cornerstone of management. Adjunctive corticosteroid therapy may be beneficial (Sharma et al., 2005; Lim and Chong, 1999).

Empirical treatment for possible but not yet definitive miliary TB increases the likelihood of survival and should never be withheld while test results are pending (Moon, 1997).

Anti-TB therapy can reduce morbidity and mortality but it may be initiated empirically (Moon, 1997).

\section{REFERENCES}

Carrol, E.D., J.E. Clark and A.J. Cant, 2001. Nonpulmonary tuberculosis. Paediatr. Respir. Rev., 2: 113119. DOI: $10.1053 /$ prrv.2000.0118

Dagli, C.E., E. Guler, V. Bakan, N. Atilla and N. Koksal, 2009. Miliary tuberculosis accompanying paravertebral tuberculosis abscess in an adolescent. J. Infect. Dev. Ctries, 3: 402-404. PMID: 19759512
Engin, G., B. Acuna, G. Acuna and M. Tunaci, 2000. Imaging of extrapulmonary tuberculosis. Radiographics, 20: 471-488. PMID: 10715344

Golden, M.P. and H.R. Vikram, 2005. Extrapulmonary tuberculosis: An overview. Am. Fam. Physician, 72: 1761-1768. PMID: 16300038

Lim, K.H. and K.L. Chong, 1999. Multiple organ failure and septic shock in disseminated tuberculosis. Singapore Med. J., 40: 176-178. PMID: 10402899

Mehta, J.B., A. Dutt, L. Harvill and K.M. Mathews, 1991. Epidemiology of extrapulmonary tuberculosis. A comparative analysis with preAIDS era. Chest, 99: 1134-1138. DOI: 10.1378/chest.99.5.1134

Moon, M.S., 1997. Tuberculosis of the spine, Controversies and a new challenge. Spine, 22: 17911797. DOI: 10.1097/00007632-199708010-00022

Sharma, S.K., A. Mohan, A. Sharma and D.K. Mitra, 2005. Miliary tuberculosis: New insights into an old disease. Lancet Infect. Dis., 5: 415-430. DOI: 10.1016/S1473-3099(05)70163-8

Yao, D.C. and D.J. Sartoris, 1995. Musculoskeletal tuberculosis. Radiol. Clin. North Am., 33: 679-689. PMID: 7610238

Zaeeter, W., A. Jathavedam and R. Stahl, 2010. A 30 year old man with fever and indolent soft tissue masses. Am. J. Infect. Dis., 6: 57-60. DOI: 10.3844/ajidsp.2010.57.60 